\title{
Biological Applications of Near-field Scanning Optical Microscopy
}

\author{
M.H.P. Moers, A.G.T. Ruiter, A. Jalocha \& N.F. van Hulst \\ Applied Optics group, Faculty of Applied Physics \& MESA Research Institute, \\ University of Twente, P.O.Box 217, 7500AE Enschede, the Netherlands. \\ W.H.J. Kalle, J.C.A.G. Wiegant \& A.K. Raap \\ Dept. of Cytochemistry and Cytometry, University of Leiden, \\ Wassenaarseweg 72, 2333AL Leiden, the Netherlands
}

\begin{abstract}
Near-field Scanning Optical Microscopy (NSOM) is a true optical microscopic technique allowing fluorescence, absorption, reflection and polarization contrast with the additional advantage of nanometer lateral resolution, unlimited by diffraction and operation at ambient conditions. NSOM based on metal coated adiabatically tapered fibers, combined with shear force feedback and operated in illumination mode, has proven to be the most powerful NSOM arrangement, because of its true localization of the optical interaction, its various optical contrast possibilities and its sensitivity down to the single molecular level. In this paper applications of "aperture" NSOM to Fluorescence In Situ Hybridization of human metaphase chromosomes are presented, where the localized fluorescence allows to identify specific DNA sequences. All images are accompanied by the simultaneously acquired force image, enabling direct comparison of the optical contrast with the sample topography on nanometer scale, far beyond the diffraction limit. Thus the unique combination of high resolution, specific optical contrast and ambient operation offers many new direction possibilities in biological studies.
\end{abstract}

\section{INTRODUCTION}

Optical microscopy and spectroscopy are still key techniques of observation in medicine and biology, because of several advantages: (i) Non-invasive, non-destructive and safe: most biological samples can be studied in their native environment (in vivo); (ii) All materials absorb, reflect, scatter light and have spectroscopic states, each giving contrast; (iii) Spectroscopy: high resolution for the chemical state; (iv) High time resolution, reaching the femtosecond time domain; (v) High sensitivity: better than 1 photon/sec. Yet in optical microscopy a natural limit is encountered: the wavelength. It is fundamentally impossible to focus light to an area smaller than this size, the diffraction limit. Consequently the spatial resolution in conventional optical microscopy is limited to about half the wavelength. This is where near-field optical microscopy comes in [1,2].

In near-field optical microscopy a miniature optical probe, either a source or detector, is scanned over a sample surface at nanometer distance. In practice the probe size is rather 10 to $100 \mathrm{~nm}$ and merely serves as a constriction to funnel an incident propagating wave to sub-wavelength dimensions, while the outgoing far field is detected after tunneling through probe and sample. The throughput of the optical system is generally low, currently $10^{-5}$ at best. Therefore far field effects must be reduced in order to observe only near field interactions. The most generally applied near field optical probe consists of a small aperture $(\sim 50 \mathrm{~nm})$ at the end of a metal coated tapered optical fiber. Since 1990 considerable progress has been made with the achievement of adiabatic fiber pulling, as introduced by Betzig et al. [3] and shear force feedback (Toledo-Crow et al. [4], Betzig et al. [5]). This combination has resulted in a reproducible, relatively efficient aperture probe, which can be operated nondestructively. Using these probes application of near field fluorescence microscopy to biological and chemical samples has been explored by Betzig et al. [6], Moers et al. [7] and Pylkki et al. [8]. In this paper we discuss recent results on fluorescence in situ hybridized chromosomes using combined aperture type near field optical and force microscopy. 


\section{MICROSCOPE SET-UP}

The configuration of an aperture NSOM operating in transmission is schematically shown in Figure 1. The near field aperture probe is fabricated by adiabatic tapering of an optical fiber using a commercially available fiber puller (Sutter P2000) and subsequent directional coating with aluminum. Thus a $50-100 \mathrm{~nm}$ aperture is created, with surrounding aluminum for screening of the far field. The probe has a brightness of $100 \mathrm{pW}-1 \mathrm{nW}$ when $\sim 5 \mathrm{~mW}$ laser light is coupled into the fiber, i.e. an efficiency of $\sim 10^{-7}$.

For biological applications it is important to design the near field microscope such that object glasses can be accommodated and the sample can be viewed with conventional high magnification optics for localization of a specific area of interest. Our near field optical microscope [9] is based on a Zeiss Axiovert inverted microscope, which was chosen for its high mechanical stability. The commercial sample table was replaced by a home built table with mechanical and electrical sample translation using piezo-electric elements with $7 \mu \mathrm{m}$ scan range. High NA objective ( $0.75 \mathrm{NA}$ dry or $1.4 \mathrm{NA}$ immersion) are used for sufficient magnification and especially efficient collection of fluorescence. Several exit ports are accessible, accommodating eyepieces, a CCD camera and a point detector. For fluorescence detection a dichroic mirror and long-pass filter are used, which block the excitation light. In near field operation the probe source is confocally imaged onto the point detector. For high light levels, $>1 \mathrm{fW}$, a photo-multiplier tube is used in combination with a $200 \mu \mathrm{m}$ pinhole in the image plane. For low light levels, up to $10^{6}$ photocounts/sec, a $100 \mu \mathrm{m}$ area photon counting avalanche photo-diode [9] is used with $\sim 59 \%$ quantum efficiency at $\lambda=600 \mathrm{~nm}$ and $\sim 9$ dark counts/sec.

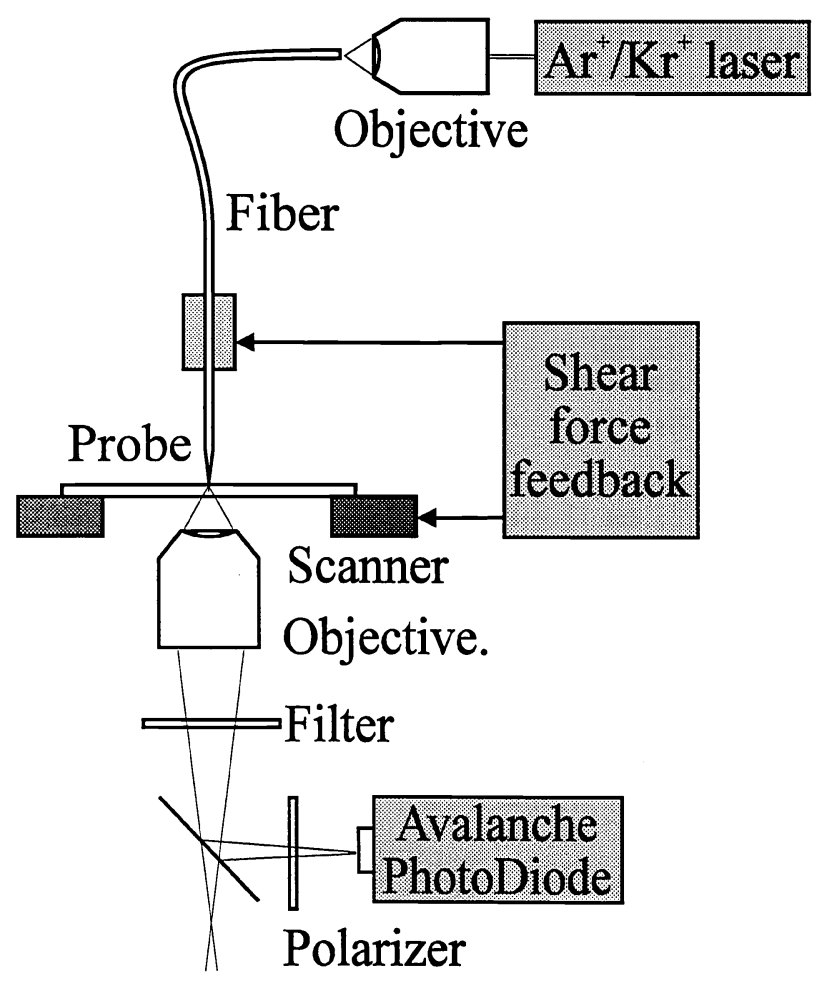

Figure 1.

Schematic set-up of the aperture type Near-field Scanning Optical Microscope (NSOM) with metallized adiabatically tapered fiber, scanned sample stage, shear force feedback on tip sample distance, and detection in transmission using a high NA objective. 
When approaching the aperture probe towards the sample surface the distance is adjusted at about $3 \mathrm{~nm}$ by a feedback system based on shear force detection [4,5]. Hereto the fiber probe is attached to a piezo-electric element which oscillates the fiber in the lateral direction (parallel to the sample surface) with an amplitude of about $20-30 \mathrm{~nm}$ at its resonance frequency (typically $>10 \mathrm{kHz}$ ). The oscillation amplitude is measured with a sensitivity of $\sim 1 \mathrm{~nm}$ by illuminating the fiber with a laser diode $(\lambda=780 \mathrm{~nm})$ and detecting the far field diffraction pattern on a split detector, where the difference signal is a measure for the fiber amplitude. On approaching the sample surface the difference signal decreases due to "shear" forces between tip and sample, which allows the operation of a feedback loop at an effective shear force value (set point). The correction signal is fed to the z-piezo-electric element in the scanner and gives simultaneously the sample height.

Near field optical, shear force and height signal are digitized and stored in a personal computer, which also generates the scan pattern and timing for photon counting.

\section{FLUORESCENCE IN SITU HYBRIDIZATION OF HUMAN CHROMOSOMES}

Over the last decade the technique of fluorescence in situ hybridization (FISH) has developed as one of the major cytogenetic detection methodologies for human genetics [10, 11]. Using fluorescent labels, FISH enables direct visualization of topological or positional information of gene sequences under a fluorescence microscope. Thus FISH allows rapid localization of genomic DNA fragments and identification of chromosomes with a superior resolution and signal-to-noise ratio compared to radioactive in situ hybridization and chromosome banding techniques. Depending on DNA condensation a resolution better than 10 basepairs can be obtained using (pro)metaphase chromosomes [12]. Yet the localization of the fluorescence labels is fundamentally limited by diffraction in conventional fluorescence microscopy. Recently Putman et al. [13] have shown that further improvement can be obtained using force microscopy to detect the morphological features of the in situ hybridization label after enhancement to $100-200 \mathrm{~nm}$ by an enzymatic cytochemical reaction. Yet fluorescence detection has the advantage of higher specificity and moreover multicolor labeling can be applied [12]. We show that the superior lateral resolution of near field fluorescence allows improved localization of the labels while maintaining the potential of multicolor labeling, provided the labels are sufficiently close to the chromosomal surface and the fluorescence level is still detectable.

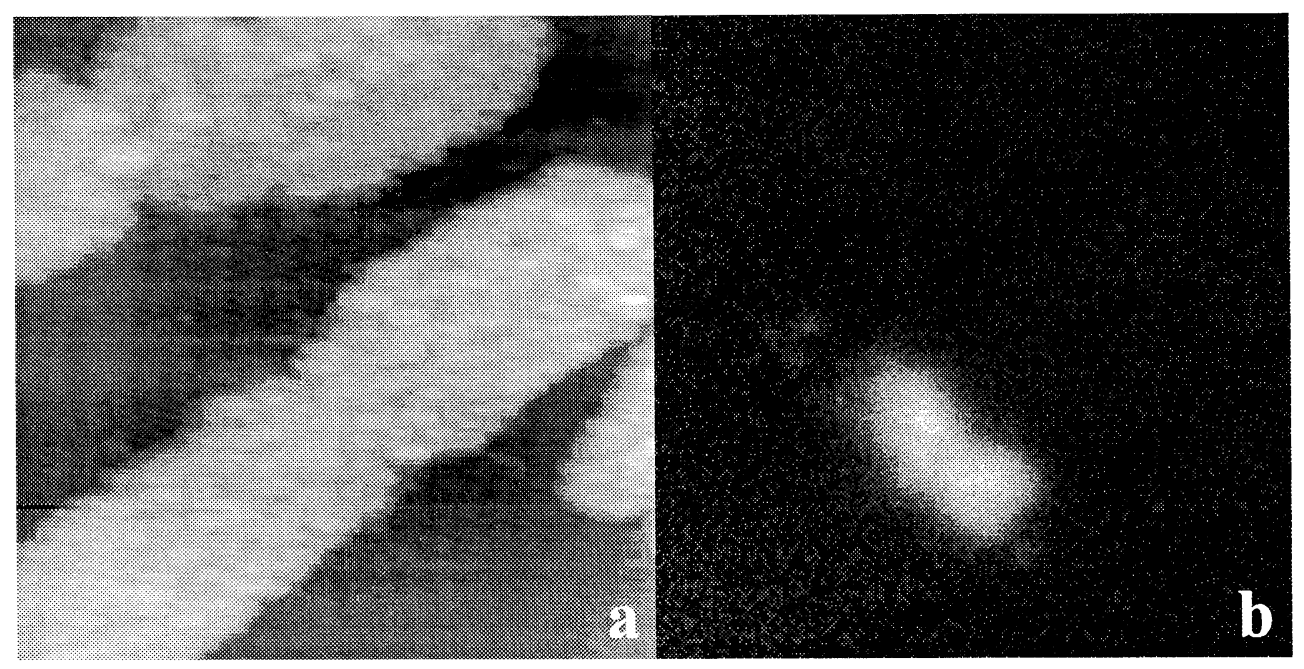

Figure 2.

A $7 \times 7 \mu \mathrm{m}^{2}$ scan of human metaphase chromosome \#1: (a) Shear force image, high pass filtered in horizontal direction; (b) corresponding near field fluorescence image displaying specific labeling of the centromeric area with CY3 fluorophore by in situ hybridization. 
Figure 2 shows a scan of human metaphase chromosome \#1 with specific labeling of the centromeric area by in situ hybridization: pUC1.77 recombinant DNA hybridized to 1q12, stained with CY3 fluorophore. In the shear force image (Fig. 2a) the high spatial frequency filtered piezo feedback signal is displayed, which shows the chromosome topography with some substructure. The corresponding near field fluorescence image (Fig. 2b) displays the green fluorescence at $\lambda>570 \mathrm{~nm}$, using BG39 and KV550 Schott filters, with excitation by the 521 $\mathrm{nm} \mathrm{Kr}{ }^{+}$-line. The image is $200 \times 200$ pixels with $25 \mathrm{msec} /$ pixel. The speckled background in the fluorescence image reflects the discrete level of 0 to 3 counts/pixel. The specific labeling of the centromeric chromosome area is clearly visible with 60 to 120 counts/pixel fluorescence. Beside the chromosome are some locally fluorescent areas due to unbound fluorophore. Based on the signal level ( $\leq 10$ counts/pixel) we estimate $\sim 10$ fluorescent molecules to be present in these spots.

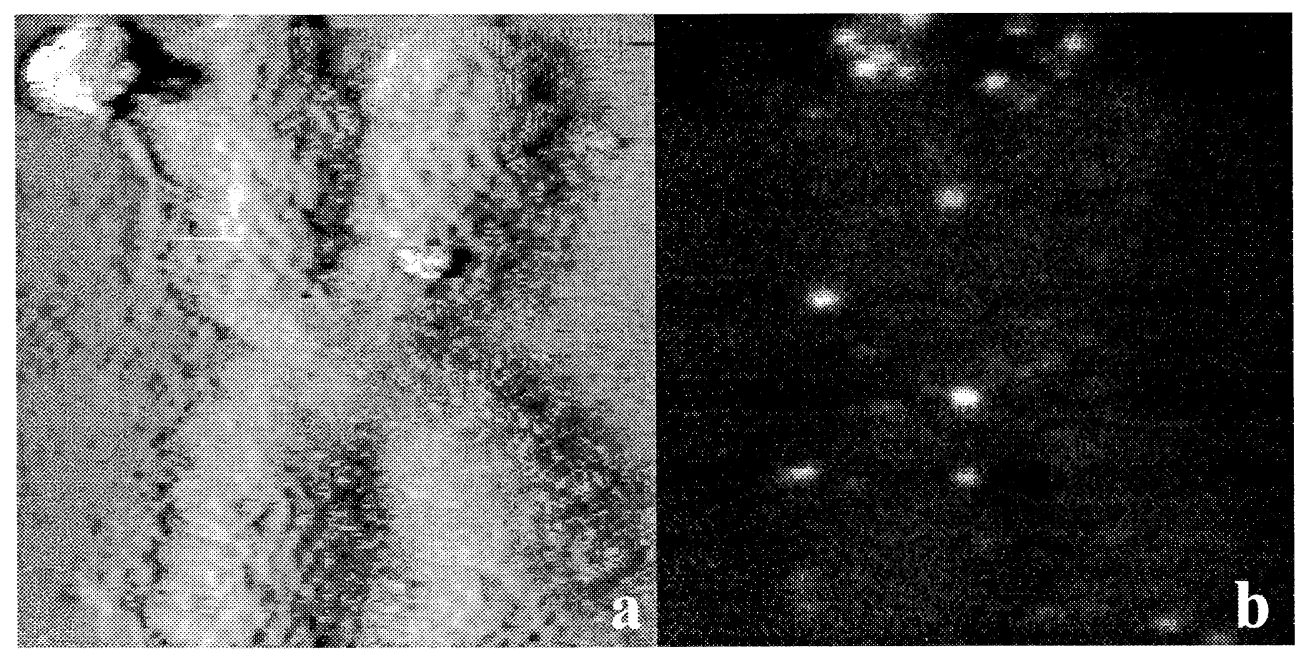

Figure 3.

A $7 \times 7 \mu m^{2}$ scan of human metaphase chromosome \#1 [14]: (a) Shear force image, high pass filtered in horizontal direction; (b) corresponding near field fluorescence image showing fluorescence in situ hybridization labels: mainly CY3 fluorescence at the telomer probe pl-79 (top) and several additional probes widely distributed over the chromatides.

Figure 3 shows another scan of a human metaphase chromosome \#1, with specific labeling of the telomeric region (top of the image) of the short arm [14]: p1-79 hybridized to 1p36, again with CY3 staining. The pixel size is $35 \mathrm{~nm}$. The scan speed is $40 \mathrm{msec} /$ pixel. The shear force image (Fig. 3a) displays the well known metaphase chromosomal structure with well separated chromatides, details as small as $75 \mathrm{~nm}$ and additional topographic structures surrounding the chromosome. The corresponding near field fluorescence image (Fig. 3b), with $521 \mathrm{~nm} \mathrm{Kr}^{+}$-line excitation and $\lambda>570 \mathrm{~nm}$ detection, shows distinct substructure in the telomeric labels (at least 5 probes in each chromatide, with maximum 700 counts/pixel) and several isolated spots both on the chromatides and in the centromeric region. The width of the fluorescence spots is less than $100 \mathrm{~nm}$, while spots only $125 \mathrm{~nm}$ separated are individually detected. Autofluorescence can be recognized as a weak general background signal over the total chromosome area. The combined images (force and fluorescence) allow accurate determination of the probe location on the chromosome structure. 
Finally figure 4 shows a scan of metaphase chromosome \#1 which has been labeled simultaneously by a centromeric morphological probe, pUC1.77 detected by the PO/DAB reaction, and a telomeric fluorescent probe, p1-70 visualized by CY-3 fluorophore. The fluorescent probe shows considerable sub-structure, indicating multiple labeling, which cannot be resolved by far-field optical techniques. Comparison of the corresponsing force and fluorescence images clearly shows the superior selectivity of NSOM over AFM.

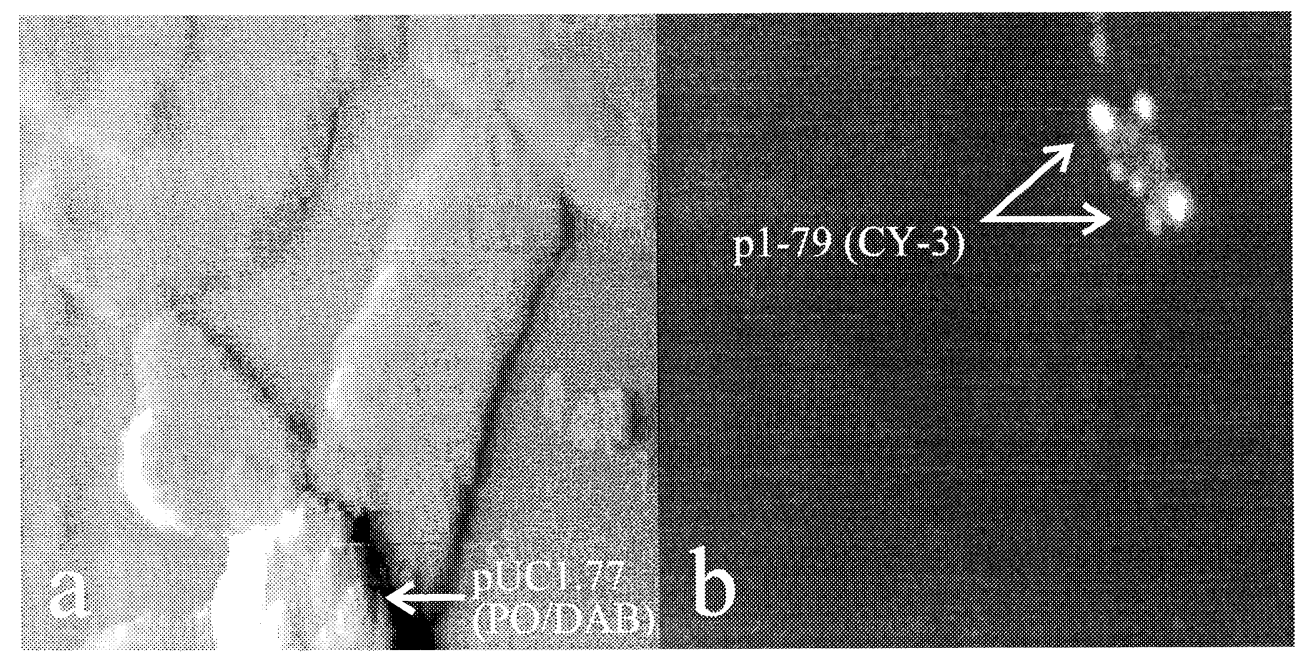

Figure 4.

A $7 \times 7 \mu \mathrm{m}^{2}$ scan of human metaphase chromosomes: (a) Shear force image, high pass filtered in horizontal direction showing the topographic pUC1.77 (PO/DAB) label; (b) corresponding near field fluorescence image showing the fluorescent pl-79 (CY-3) label and slight auto-fluorescence.

\section{CONCLUSIONS}

We have presented biological applications of near field optical microscopy, in combination with force microscopy. Aperture NSOM with fluorescence detection gives (bio)chemical specificity, in addition to the simultaneously acquired force image, which has large potential for DNA sequencing. Fluorescence NSOM gives high resolution on flat not too deep surfaces. Fluorescence NSOM induces virtually no bleaching as opposed to confocal fluorescence microscopy. Shear force feedback is essential in aperture NSOM operation with fibers and operates on soft surfaces of cells and chromosomes. Ultimately aperture NSOM is limited by its low efficiency with a source brightness of typically $100 \mathrm{pW}$ to $10 \mathrm{nW}$. Thus in spectroscopic applications (fluorescence, Raman, etc.) photon noise will be a fundamental limit in the speed of imaging. Clearly the virtues of optics, noninvasiveness, high spectral resolution and high time resolution, all apply to the near field optical domain with its high spatial resolution, which adds extensively to the potential of scanning probe microscopy Thus application potential of NSOM in biology is obvious.

\section{ACKNOWLEDGMENTS}

The authors thank Kees van der Werf, Frans Segerink, Eric Schipper, Ine Segers and Bart de Grooth for their assistance and suggestions. This research is supported by the Dutch Foundation for Fundamental Research (FOM) and the European network on Near-field Optics and Nanotechnology. 


\section{REFERENCES}

1. Pohl DW, Novotny L: Near-field optics: light for the world of nano. J Vac Sci Technol B 12: 1441-1446, 1994.

2. For a recent overview of the field of "Near-field optics" see, Proc. 2nd International Conference on Nearfield Optics, Raleigh, NC, oct 1993, ed. M. Isaacson, Ultramicroscopy, 57: vol. 2 \& 3, 1995.

3. Betzig E, Trautman JK: Near-field optics: microscopy, spectroscopy and surface modification beyond the diffraction limit. Science 257: 189-195, 1992.

4. Toledo-Crow R, Yang PC, Chen Y, Vaez-Iravani M: Near-field differential scanning optical microscope with atomic force regulation. Appl Phys Lett 60: 2957-2959, 1992.

5. Betzig E, Finn PL, Weiner JS: Combined shear force and near-field scanning optical microscopy. Appl Phys Lett 60: 2484-2486, 1992.

6. Betzig E, Chichester RJ: Near-field Fluorescence Imaging of Cytoskeletal Actin. Bioimaging 1: 129-133, 1993.

7. Moers MHP, Gaub HE, Van Hulst NF: Poly(diacetylene) monolayers studied with a fluorescence scanning near-field optical microscope. Langmuir 10: 2774-2777, 1994.

8. Pylkki RJ, Moyer PJ, West PE: Scanning near field optical microscopy and scanning thermal microscopy. Jpn J Appl Phys 33: 3785-3790, 1994.

9. Moers MHP, Ruiter AGT, Van Hulst NF, Bölger B: Optical contrast in near-field techniques. Ultramicroscopy 57: 298-302, 1995.

10. Rudkin GT, Stollar BD: High resolution detection of DNA-RNA hybrids in situ by indirect immunofluorescence Nature 265: 472-473, 1977.

11. Bauman JGJ, Wiegant J, Van Duijn P: Cytochemical hybridisation with fluorochrome-labeled RNA. $J$ Histochem Cytochem 29: 227-246, 1981.

12. Wiegant J, Wiesmeijer CC, Hoovers JMN, Schuuring E, d'Azzo A, et al: Multiple and sensitive fluorescence in situ hybridisation with rhodamine-, fluorescein- and coumarin-labelled DNAs. Cytogenetics and Cell Genetics 63: 73-76 1993.

13. Putman CAJ, De Grooth BG, Wiegant J, Van der werf KO, Van Hulst NF, et al: Detection of in situ hybridization to human chromosomes with the atomic force microscope. Cytometry 14: 356-361, 1993.

14. Moers MHP, Kalle WHJ, Raap AK, De Grooth BG, Van Hulst NF, et al: Fluorescence in situ hybridisation on metaphase chromosomes observed by near-field microscopy. J Microscopy, to be pub. 1995. 